\title{
Anomalous effective action, Noether current, Virasoro algebra and Horizon entropy
}

\author{
Bibhas Ranjan Majhi ${ }^{1,2, a}$, Sumanta Chakraborty ${ }^{1, b}$ \\ ${ }^{1}$ IUCAA, Ganeshkhind, Pune University Campus, Post Bag 4, Pune 411 007, India \\ ${ }^{2}$ Racah Institute of Physics, Hebrew University of Jerusalem, Givat Ram, 91904 Jerusalem, Israel
}

Received: 11 February 2014 / Accepted: 22 April 2014 / Published online: 20 May 2014

(C) The Author(s) 2014. This article is published with open access at Springerlink.com

\begin{abstract}
Several investigations show that in a very small length scale there exist corrections to the entropy of black hole horizon. Due to fluctuations of the background metric and the external fields the action incorporates corrections. In the low energy regime, the one-loop effective action in four dimensions leads to trace anomaly. We start from the Noether current corresponding to the Einstein-Hilbert plus the one-loop effective action to calculate the charge for the diffeomorphisms which preserve the Killing horizon structure. Then a bracket for the charges is calculated. We show that the Fourier modes of the bracket are exactly similar to the Virasoro algebra. Then using the Cardy formula the entropy is evaluated. Finally, the explicit terms of the entropy expression is calculated for a classical background. It turns out that the usual expression for the entropy; i.e. the BekensteinHawking form, is not modified.
\end{abstract}

\section{Introduction and motivation}

One of the striking features of general relativity is its deep connection with the laws of thermodynamics. A "marriage" between the general theory of relativity (GR) and quantum mechanics shows that black holes behave as thermodynamic objects with a Hawking temperature $T_{H}=\kappa / 2 \pi$ and an intrinsic entropy given by the Bekenstein-Hawking area law, $S=A_{h} / 4 G$, where $\kappa$ is the surface gravity and $A_{h}$ is the area of the horizon [1-5] (for recent reviews see [6,7]). It should be noted that the interpretation of classical black hole laws in terms of black hole thermodynamics has become possible due to taking into account quantum mechanical effects, leading to Hawking radiation. Also we could readily obtain the

\footnotetext{
a e-mail: bibhas.majhi@mail.huji.ac.il; bibhas@iucaa.ernet.in

be-mail: sumanta@iucaa.ernet.in
}

same area dependence of entropy and that leads to a kind of universality for the above result.

Although thermodynamic interpretations were first observed in black hole solutions, such features are much more general. It has been observed that the accelerated observer on a Minkowski spacetime also associates temperature and entropy on the Rindler horizon. This originates with the Unruh effect [8]. This leads us to think that the static observer (black hole) and the Rindler observer (accelerated frame) are to be treated on the same footing. The reality is much more general. In a local region one can always have a null surface which is not a solution of the Einstein equation and on which an entropy functional can be associated such that evaluation of it on the null surface leads to the entropy expression. Moreover, the extremization of it leads to the Einstein equations. This is very important in the context of the emergent paradigm of gravity [9]. Such a local concept implies that the notion of entropy is much more fundamental and that it must be an observer-dependent quantity. Hence any analysis leading to it must have an observer-dependent off-shell description.

For the more general class of theories including higher curvature terms in the action the Bekenstein-Hawking area law no longer applies. However, assuming the zeroth law, the first law can be derived in this class of covariant actions [10]. Here the Noether charge corresponding to the diffeomorphism symmetry, calculated over the spatial cross-sections of the horizon, plays the role of entropy. This mode of thinking has two important aspects, which are as follows:

- These results imply that the connection between gravity and thermodynamics is valid well beyond the Einstein theory of gravity. It only requires some general argument, like covariance and the principle of equivalence (In some modified gravity theories without the equivalence principle one can construct black hole solutions, first laws, and thermal radiation $[11,12])$. 
- It is also possible to attribute an observer-dependent entropy to any null surfaces (which need not be event horizons).

So far the results are well valid in the classical regime. On a very small length scale, like the Planck length, quantum effects have to be incorporated, particularly since the fluctuations of the metric and the external fields cannot be ignored. Then the classical results have to be modified. One way to include these effects is to find the effective action corresponding to the fluctuations. It modifies the partition function and hence the entropy [13]. It turns out that in GR the leading order correction to the entropy is logarithmic in the horizon area [14] and the coefficient of it is related to the trace anomaly. Similar results have been achieved in several methods (see for example [15-21]), but the value of the coefficient depends on the particular model for calculating the entropy. On the other hand, the entanglement of quantum fields between inside and outside of horizon approach leads to power law corrections [22]. Hence it is evident that the existence of corrections is universal and one would expect an identical situation in a method of computing the entropy.

One of the interesting ways to understand this universality is by the Noether charge prescription. This will be done for the anomalous effective action due to the fluctuations of the quantum fields, which leads to the trace anomaly in four dimensions. The one-loop effective action can be determined by the effective field theory technique. In the low energy limit, if one breaks the conformal symmetry the resulting theory leads to the trace anomaly [23-26]. In [27,28], an attempt has been done by evaluating the Noether charge corresponding to the GR action plus this one-loop effective action for a Killing vector. It must be noted that the charge was derived by using the equation of motion and so the analysis is on-shell. Here we will present a completely off-shell analysis.

So far no attempts have been made to quantify the degrees of freedom which are responsible for such a universality. This issue we will discuss in the context of the Virasoro algebra and the Cardy formula. The method was first introduced in the context of gravity by Brown and Hannueax [29] and later developed by Carlip [30]. Although the method has been followed up in several gravity theories [31-35], no discussion exists of this in the presence of the one-loop effective action which leads to the trace anomaly. Here we will fill up this blank. Our analysis will be followed from a recent work of one of the authors [35]. The key features of the calculation are:

(i) The whole analysis will be off-shell; , i.e. no equation of motion will be used. (ii) The derivation of the current corresponding to the total action (i.e. the Einstein-Hilbert part plus the one-loop effective action) is off-shell and it is off-shell conserved.

These are essential since, as we have argued earlier, the notion of entropy has a general sense beyond the concept of the black hole horizon.

Let us first summarize the methodology. The main step is to define a bracket among the charges. In a previous work of one of the authors [35], an off-shell definition of the bracket has been given for any general covariant Lagrangian in terms of the arbitrary diffeomorphism vector and Noether current. Here we will use this definition where an explicit form of the current will be taken for the present theory. To evaluate it the diffeomorphism vectors will be chosen using Carlip's formalism [30]. This essentially tells that the vectors are chosen in such a way that they are asymptotically Killing vectors near the horizon so that the asymptotic Killing horizon remains invariant. It turns out that the Fourier modes of the bracket are similar to the standard form of the Virasoro algebra which has a central extension. Then the central charge and the zero mode eigenvalue are automatically identified. Substituting these in the Cardy formula [36,37] we obtain the expression for the entropy. We will show that the entropy will incorporate no correction for a classical background to the usual Bekenstein-Hawking expression. Physically this analysis tells that some of the degrees of freedom (DOF), which were originally gauge DOF, are raised to true DOF, which leads to an entropy. Since this is happening due to the imposition of a particular condition on the diffeomorphisms, the DOF responsible for the entropy are observer dependent. This has been elaborated earlier in more detail in [32-34].

The organization of our paper is as follows. In Sect. 2 we present an analysis for a general covariant Lagrangian. First the form of the Noether charge for a general covariant Lagrangian is given. Next we give the expressions for the Fourier modes of the charge and the central term corresponding to the diffeomorphisms which keep the Killing horizon structure invariant and are asymptotically Killing vector near the horizon. Section 3 is devoted to an explicit calculation of these quantities for the anomalous effective action. We then find the entropy using the Cardy formula. Finally, we conclude in Sect. 4. For completeness, an appendix has been given at the end of the paper.

\section{Virasoro algebra and central term: a general approach}

The recent progress in black hole thermodynamics shows that the microscopic features may not be too sensitive to the details of quantum gravity as the derivations for black hole 
temperature and entropy only use semiclassical gravity. In order to achieve this it was first pointed out by Strominger that a quantum state calculation should rely on symmetry [38]. In an earlier work, Brown and Henneaux [29] showed that $(2+1)$-dimensional gravity has an asymptotic symmetry consisting of a Virasoro algebra with central extension, implying that any microscopic quantum theory should be a conformal field theory. Strominger noticed that the use of the above central charge in the Cardy formula [36,37] leads to the usual Bekenstein-Hawking entropy. This implies that the asymptotic symmetries might shed some light on the calculation of the density of states, leading to the statistical description of entropy. The limitations of the earlier works are that they use the asymptotic AdS symmetry, which is insensitive to the structure of interior spacetime; i.e. the details of the horizon. Moreover, the method is confined to $(2+1)$ dimensions. Later on, Carlip introduced an approach which relies on the Killing horizon structure preserving diffeomorphisms and which is independent of the dimension of the spacetime [30]. So it reflects the information of the horizon which is important because the entropy depends on the structure of the horizon. In this paper, we shall adopt this approach.

In this section, we shall present the general expressions for the Fourier modes of the Noether charge $Q$ and the central term corresponding to a generally covariant Lagrangian. This method not only applies to Einstein gravity but also to any theories of gravity whose Lagrangian is a function of the metric, the curvature tensor, and some other external fields, like a scalar field. The technique we shall follow is from [30,35].

Let us first introduce the general expressions for the Noether conserved current $J^{a}$ and the charge $Q$ corresponding to a generally covariant Lagrangian. The explicit variation of this general Lagrangian for the metric variation leads to the following form:

$\delta\left(L_{\text {grav }} \sqrt{-g}\right)=\sqrt{-g}\left\{E_{a b} \delta g^{a b}+\nabla_{a}\left(\delta v^{a}\right)\right\}$

where $E_{a b}=0$ leads to the equation of motion and $\delta v^{a}$ is the surface term. Now if the variation is given by the Lie derivative due to the coordinate transformation $x^{a} \rightarrow x^{a}+\xi^{a}$, then $\delta g_{a b} \equiv £_{\xi} g_{a b}=\nabla_{a} \xi_{b}+\nabla_{b} \xi_{a}$. Therefore, using the generalized Bianchi identity $\nabla_{a} E^{a b}=0$ [9], the first term on the right hand side of Eq. (1) can be cast into a total derivative form, $-2 \sqrt{-g} \nabla_{a}\left(E_{b}^{a} \xi^{b}\right)$. On the other hand, since $L_{\text {grav }} \sqrt{-g}$ density, the Lie variation is given by $\sqrt{-g} \nabla_{a}\left(L_{\text {grav }} \xi^{a}\right)$. Using all these in Eq. (1) we obtain a conservation relation given by $\nabla_{a} J^{a}=0$, where

$J^{a}=\left(L_{\text {grav }} \xi^{a}-£_{\xi} v^{a}+2 E^{a b} \xi_{b}\right)$.

Here $£_{\xi} v^{a}$ represents a boundary term arising from the Lie variation of the metric as stated earlier. The current $J_{a}$ is called the Noether current. Since the current is covariantly conserved, it can be expressed as the covariant derivative of an antisymmetric tensor: $J^{a}=\nabla_{b} J^{a b}$, where $J^{a b}$ is known as the Noether potential. For the general class of covariant gravitational theories, substituting the respective values in (2) one can obtain the explicit expression. This leads to the current and potential as (see page 394 of [39] for details):

$$
\begin{aligned}
J^{a}= & \frac{1}{8 \pi G} P^{a b c d} \nabla_{b} \nabla_{c} \xi_{d}-\frac{1}{8 \pi G} \nabla_{b}\left(P^{a d b c}+P^{a c b d}\right) \\
& \times \nabla_{c} \xi_{d}-\frac{1}{4 \pi G} \xi_{d} \nabla_{b} \nabla_{c} P^{a b c d}, \\
J^{a b}= & \frac{1}{8 \pi G} P^{a b c d} \nabla_{c} \xi_{d}-\frac{1}{4 \pi G}\left(\nabla_{c} P^{a b c d}\right) \xi_{d}
\end{aligned}
$$

where the four index tensor $P^{a b c d}$ is defined as $P^{a b c d}=$ $\partial L_{\text {grav }} / \partial R_{\text {abcd }}$. This tensor has identical symmetry properties to that of the curvature tensor $R^{a b c d}$, i.e. it is antisymmetric under interchange of $a, b$ and $c, d$, and symmetric under interchange of pairs $(a, b)$ and $(c, d)$ along with $P^{a(b c d)}=0$. The corresponding Noether charge is defined as

$Q[\xi]=\frac{1}{2} \int d \Sigma_{a b} \sqrt{h} J^{a b}$

where $d \Sigma_{a b}$ corresponds to the surface element. For instance, using Eq. (4) one can show that the explicit expression for the Noether potential corresponding to Einstein gravity appears to be $J^{a b}=(1 / 16 \pi G)\left(\nabla^{a} \xi^{b}-\nabla^{b} \xi^{a}\right)$. Now using this in Eq. (5) for a timelike Killing vector field $\xi^{a}=\chi^{a}$, the Noether charge $Q$, calculated on the horizon, can be shown to be related to the horizon entropy. More specifically, $Q$ multiplied by the periodicity of the Euclidean time leads to the Bekenstein-Hawking entropy expression: $(2 \pi / \kappa) Q=A_{h} / 4 G$, with $\kappa$ being the surface gravity. The above procedure has also been extended for Lanczos-Lovelock models to get the black hole entropy as the Noether charge [35]. However, note that in all these models we have $\nabla_{a} P^{a b c d}=0$. Having set the stage we shall now deal with the general expressions (3) and (4) to consider the situation where $\nabla_{a} P^{a b c d} \neq 0$.

Next we give the expressions for the Fourier modes of the charge and the central extension. Applying the method of Carlip [30] for a stretched horizon scenario and using the definition of the bracket among the charges, given in [35], we find the Fourier modes of the charge as

$$
\begin{aligned}
Q_{m}= & -\frac{1}{32 \pi G} \int \sqrt{h} d^{d-2} X P^{a b c d} \mu_{a b} \mu_{c d} \\
& \times\left[2 \kappa T_{m}-\frac{1}{\kappa} D^{2} T_{m}\right] \\
& -\frac{1}{8 \pi G} \int \sqrt{h} d^{d-2} X \mu_{a b} \chi_{d} T_{m}\left(\nabla_{c} P^{a b c d}\right),
\end{aligned}
$$


while the Fourier modes for the central term turn out to be

$$
\begin{aligned}
& K\left[\xi_{m}, \xi_{n}\right] \\
&=-\frac{1}{32 \pi G} \int \sqrt{h} d^{d-2} X P^{a b c d} \mu_{a b} \mu_{c d} \frac{1}{\kappa} D T_{m} D^{2} T_{n} \\
&-\frac{1}{8 \pi G} \int \sqrt{h} d^{d-2} X \frac{|\chi|}{\rho} \frac{\chi_{b} \chi_{c} \rho_{d}}{\kappa \chi^{2}} T_{n} D^{2} T_{m} \nabla_{f} P^{b c f d} \\
&-\frac{1}{8 \pi G} \int d^{d-2} X \sqrt{h} \frac{|\chi|}{\kappa^{2} \chi^{2} \rho} \chi_{b} \chi_{c} \rho_{d} D T_{n} D^{2} T_{m} \nabla_{f} P^{b d f c} \\
&+\frac{1}{4 \pi G} \int d^{d-2} X \sqrt{h} \frac{|\chi|}{\kappa \rho^{3}} \\
& \times\left(\chi^{2} \rho_{b} \rho_{d}-\rho^{2} \chi_{b} \chi_{d}\right) T_{n} D T_{m} \nabla_{f} \nabla_{c} P^{b f c d} \\
&-\frac{1}{4 \pi G} \int \sqrt{h} d^{d-2} X \frac{|\chi|}{\rho \chi^{2}} \chi_{a} \rho_{b} \chi_{d} T_{m} D T_{n} \nabla_{c} P^{a b c d} \\
&-\frac{1}{4 \pi G} \int \sqrt{h} d^{d-2} X \frac{|\chi|}{\rho \chi^{2}} \\
& \times\left(\frac{\chi^{2}}{\kappa \rho^{2}}\right)^{2} D T_{m} D^{2} T_{n} \chi_{a} \chi_{b} \rho_{d} \nabla_{c} P^{a b c d}-(m \leftrightarrow n) . \quad \text { (7) }
\end{aligned}
$$

The detailed derivation of the above quantities can be taken from [35]. But for completeness and clarity, we have shown this in Appendix A. We will use these expressions to identify the zero mode eigenvalue and the central charge to calculate the entropy. To evaluate the above, the explicit expression for $T_{m}$ is needed. This will be chosen subject to the condition that the Fourier modes for the diffeomorphism vector $\xi^{a}$ obey the following subalgebra isomorphic to Diff $S^{1}$ :

$i\left\{\xi_{m}, \xi_{n}\right\}^{a}=(m-n) \xi_{m+n}^{a}$

with $\{$,$\} being the Lie bracket. Keeping this condition in$ mind, the form for $T_{m}$ can be taken as

$T_{m}=\frac{1}{\alpha} \exp \left[i m\left(\alpha t+g(x)+p \cdot x_{\perp}\right)\right]$

where $\alpha$ is a constant and $g(x)$ is a function which is regular at the Killing Horizon. Here $p$ is an integer and $x_{\perp}$ is the $(d-2)$-dimensional transverse coordinates with the $t-x$ plane defining the null surface. The explicit expression (9) for $T_{m}$ will be needed later to evaluate the charge and the central term. However, as we will see later, this will involve the choice for the parameter $\alpha$. To obtain the correct expression for the entropy one has to consider the periodicity of the Euclidean time $2 \pi / \kappa$. Then for periodicity in the time coordinate of Eq. (9), one must have $\alpha=\kappa$. This will be needed at the end of the paper. For Lanczos-Lovelock gravity $\nabla_{c} P^{a b c d}$ vanishes and so the above expressions reduce to those obtained earlier in [35]. Here the results are much more general and can be applied for the covariant gravity theory for which the covariant derivative of $P^{a b c d}$ does not vanish. Moreover, the expressions are off-shell and free of any ambiguity, as mentioned earlier.

Having obtained these general results we shall now apply these results for anomaly-induced effective action arising from the fluctuations in the matter fields and background metric. The purpose of the next section is to calculate each of the terms of Eqs. (6) and (7) for the anomalous effective action and use them in the Cardy formula to derive the corrections to the usual form of the entropy. The analysis will be done for the case where the four-dimensional trace anomaly appears. The basic idea is that the trace anomaly, being independent of the renormalization schemes and quantum states, has some effects on the quantum correction to the black hole area law. Furthermore, the explicit expression for the anomaly can be derived from the anomaly-induced effective action. Hence it would be interesting to find the Noether charge and then use it to find the entropy. Actually, to use Eqs. (6) and (7), we need to find the explicit expression for $P^{a b c d}$ for the action on which we are interested. Then substitution of it will lead to the final result.

\section{Anomalous effective action and entropy}

In this section we shall briefly review the anomalous effective action for the fluctuations of the fields and consequently the Noether charges thus are obtained. Classical general relativity does not take into account the microscopic effects of quantum matter on any scale. In order to discuss the wavelike nature of a particle or phase correlation we need at least a semi-classical treatment of the effective stress energy tensor appearing in the Einstein equation. The one-loop effective action could be determined by the method called Effective Field Theory (EFT). It can be shown that non-local macroscopic coherence effects are actually self-contained in low energy EFT provided the one-loop trace anomaly for massless fields is included in Einstein theory [26]. The low energy EFT of gravity contains an expansion in the derivatives of the local term, while the higher order terms are suppressed by the inverse ultraviolet cutoff scale $M$. Also the theory is not renormalizable; however, this is not sensitive to all the microscopic details due to decoupling of short distance degrees of freedom [23]. When we break a classical symmetry by the quantum trace anomaly, the decoupling of short and long distance physical situations using the standard technique fails. Thus an anomaly can have a significant effect on low energy EFT. The necessity of the trace anomaly for low energy EFT of gravity can be understood from the behavior of different terms in the effective gravitational action under global Weyl rescaling. Thus the addition of anomaly terms to the 
low energy effective gravity action is consistent with both quantum theory and the equivalence principle [41].

This search for quantum corrections to the black hole entropy takes place in the context of quantum field theory in curved spacetime [42]. The backreaction of quantum fields on curved spacetime is determined by the semiclassical Einstein equation,

$G_{a b}+\Lambda g_{a b}=8 \pi\left\langle T_{a b}\right\rangle$,

such that the quantum fields affect the curved background through the expectation value of the energy-momentum tensor. This expectation value can be obtained using the oneloop correction due to the fluctuations in the quantum fields to the classical action and then taking the variation with respect to the metric. The trace of it is then given by ([25], [26])

$\langle T\rangle \equiv-\frac{2}{\sqrt{-g}} g^{a b} \frac{\delta S_{e f f}}{\delta g^{a b}}=-\frac{2}{\sqrt{-g}} g^{a b} \frac{\delta S_{\text {anom }}}{\delta g^{a b}}$.

In four dimensions there exist three geometric contributions to the anomaly-induced action, namely the Euler density (called Type-A), the Weyl tensor squared (called Type-B) and a $\square R$ term that comes from variation of $R^{2}$. Here we will pay attention only to the type-A anomaly, since it is interesting in various ways for the study of black holes [41] and also it is simple compared to other types. This type of anomaly leads to the trace anomaly:

$\langle T\rangle=-\frac{a}{16 \pi^{2} G} E_{4}$,

where $E_{4}=R_{a b c d} R^{a b c d}-4 R_{a b} R^{a b}+R^{2}$. The corresponding low energy effective action is given by

$S_{\text {eff }}=-\frac{1}{16 \pi G} \int d^{4} x \sqrt{-g} R+S_{\text {anom }}$

where the anomalous part of the action is of the form $S_{\text {anom }}=$ $S_{0}+S_{1}+S_{2}+S_{3}$ with $[27,28]$

$S_{0}=-\frac{a}{32 \pi^{2} G} \int d^{4} x \sqrt{-g}\left\{-(\square \phi)^{2}\right\}$;

$S_{1}=-\frac{a}{16 \pi^{2} G} \int d^{4} x \sqrt{-g}\left(R^{a b}-\frac{1}{3} R g^{a b}\right) \nabla_{a} \phi \nabla_{b} \phi ;$

$S_{2}=\frac{a}{48 \pi^{2} G} \int d^{4} x \sqrt{-g} \phi \square R ;$

$S_{3}=-\frac{a}{32 \pi^{2} G} \int d^{4} x \sqrt{-g} E_{4} \phi$.

The scalar field $\phi$ satisfies the following equation of motion:

$\left[\square^{2}+2 \nabla_{\mu}\left(R^{\mu \nu}-\frac{1}{3} g^{\mu \nu} R\right) \nabla_{\nu}\right] \phi=\frac{1}{2}\left(E_{4}-\frac{2}{3} \square R\right)$.

Note that $S_{0}$ does not contain any curvature part and so for that part the derivative with respect to $R_{a b c d}$ vanishes. Thus there is no Noether charge corresponding to this part. The standard way to get the trace anomaly, as first pointed out by Polyakov [24], is to find the conformal primitive. In four dimensions we need two generalizations, firstly the conformal properties of the Gaussian curvature where the d'Alembertian is determined by the Q-curvature defined as $E_{4}-\frac{2}{3} \square R$ [43], and secondly the Paneitz operator [44] should be introduced (see also $[45,46])$.

The entropy tensors can be obtained from the above actions by the usual prescription. These are given by

$$
\begin{aligned}
P_{1}^{a b c d}= & -\frac{a}{32 \pi^{2} G}\left[\left(g^{a c} \nabla^{b} \phi \nabla^{d} \phi-g^{a d} \nabla^{b} \phi \nabla^{c} \phi\right)\right. \\
& \left.-\frac{1}{3}\left(g^{a c} g^{b d}-g^{a d} g^{b c}\right)\right], \\
P_{2}^{a b c d}= & -\frac{a}{96 \pi^{2} G} \square \phi\left(g^{a c} g^{b d}-g^{a d} g^{b c}\right), \\
P_{3}^{a b c d}= & -\frac{a}{32 \pi^{2} G} \phi\left[R^{a b c d}-4\left(g^{a c} R^{b d}-g^{a d} R^{b c}\right)\right. \\
& \left.+R\left(g^{a c} g^{b d}-g^{a d} g^{b c}\right)\right] .
\end{aligned}
$$

Along with the usual Noether charge due to the EinsteinHilbert action we have three extra charges which are obtained by substitution of the respective entropy tensors in Eq. (4) and using the definition Eq. (5) for the charge. These turn out to be

$$
\begin{aligned}
Q_{E H}= & \frac{1}{16 \pi G} \int d \Sigma_{a b} \nabla^{a} \xi^{b}, \\
Q^{(1)}= & -\frac{a}{16 \pi^{2} G} \int d \Sigma_{c d} \\
& \times\left[\nabla^{c} \xi^{p} \nabla^{d} \phi \nabla_{p} \phi-\frac{1}{3}(\nabla \phi)^{2} \nabla^{c} \xi^{d}\right] \\
& + \text { terms containing }\left(\nabla_{c} P^{a b c d}\right),
\end{aligned}
$$

$$
\begin{aligned}
Q^{(2)}= & \frac{a}{48 \pi^{2} G} \int d \Sigma_{c d} \nabla^{c} \xi^{d} \square \phi \\
& + \text { terms containing }\left(\nabla_{c} P^{a b c d}\right),
\end{aligned}
$$

$$
\begin{aligned}
Q^{(3)}= & -\frac{a}{32 \pi^{2} G} \int d \Sigma_{a b}\left[R^{a b c d} \nabla_{c} \xi_{d}\right. \\
& \left.+4\left(\nabla^{a} \xi^{d} R_{d}^{b}-\nabla^{d} \xi^{a} R_{d}^{b}\right)+R \nabla^{a} \xi^{b}\right] \\
& + \text { terms containing }\left(\nabla_{c} P^{a b c d}\right) .
\end{aligned}
$$

Note that these are identical to those obtained in $[27,28]$ except the terms containing the covariant derivative of $P^{a b c d}$. However, it can be shown that these terms behave as $\mathcal{O}\left(\chi^{2}\right)$ near the horizon for $\xi^{a}$, chosen to be a timelike Killing vector $\chi^{a}$. For instance, let us consider $P_{2}^{a b c d}$, present in Eq. (19). Then $\nabla_{c} P^{a b c d}=-\left(a / 96 \pi^{2} G\right)\left(g^{a c} g^{b d}-g^{a d} g^{b c}\right) \nabla_{c} \square \phi$ as the covariant derivative of the metric tensor vanishes. Now to evaluate this term explicitly, for simplicity we take a general spherically symmetric metric of the form 
$d s^{2}=-f(r) d t^{2}+d r^{2} / f(r)+r^{2} d \Omega^{2}$. Then one can easily show that the relevant quantity $\mu_{a b} \chi_{d} \nabla_{c} P^{a b c d}$ is $\mathcal{O}(f)$ since the timelike Killing vector in this case is given by $\chi^{a}=(1,0,0,0)$. Similar conclusions hold for other parts also. Hence the terms, containing the covariant derivative of entropy tensor, in the above equations are all of $\mathcal{O}\left(\chi^{2}\right)$ as $\chi^{2}=-f$. Wald [10] was first to introduce the idea that entropy could be calculated from the Noether charge by choosing the diffeomorphism vector $\xi^{a}$ as the timelike Killing vector $\chi^{a}$. In the original formulation we have two spacetime boundaries, the asymptotic region and the horizon. The Noether charges on the asymptotic region determines the mass and the angular momentum, while that on the horizon determines the entropy. The boundary conditions are very important for the calculation of the Noether charges for they can get modified by modification of the boundary terms. Remember that, to determine the entropy, we need to calculate the charges (22), (23), (24), and (25) on the horizon defined by the relation $g_{a b} \chi^{a} \chi^{b}=0$. So the $\mathcal{O}\left(\chi^{2}\right)$ terms do not contribute and hence one can drop those terms.

Following similar arguments as above, it is also possible to show that the terms containing the covariant derivative of $P^{a b c d}$ in Eqs. (6) and (7) are of the order $\chi^{2}$. So they will not contribute near the horizon. Taking into account this fact and following identical steps to [35], we obtain from Eq. (6) and Eq. (7)

$Q_{m}=\frac{\hat{A}}{8 \pi G} \frac{\kappa}{\alpha} \delta_{m, 0}$,

$K\left[\xi_{m}, \xi_{n}\right]=-i m^{3}\left[\frac{\hat{A}}{8 \pi G} \frac{\alpha}{\kappa}\right] \delta_{m+n, 0}$

where we have introduced the Wald entropy function

$\hat{A}=-\frac{1}{2} \int \sqrt{h} d^{d-2} X P^{a b c d} \mu_{a b} \mu_{c d}$

which leads to the horizon area in GR. To obtain Eqs. (26) and (27), Eq. (9) has been used and then the integration is done over the transverse coordinates. Collecting all these it is possible to find the following form:

$i\left[Q_{m}, Q_{n}\right]=(m-n) Q_{m+n}+\frac{C}{12} m^{3} \delta_{m+n, 0}$,

which is in the form of a Virasoro algebra with central extension. $C$ is known as the central charge and is given by

$\frac{C}{12}=\frac{\hat{A}}{8 \pi G} \frac{\alpha}{\kappa}$.

The zero mode eigenvalue from Eq. (26) turns out to be

$Q_{0}=\frac{\hat{A}}{8 \pi G} \frac{\kappa}{\alpha}$.

Therefore, we obtain the central charges and zero mode eigenvalues from Eq. (30) and Eq. (31) for each term of the action as
$\frac{C^{E H}}{12}=\frac{A_{h}}{8 \pi G} \frac{\alpha}{\kappa} ; \quad Q_{0}^{E H}=\frac{A_{h}}{8 \pi G} \frac{\kappa}{\alpha} ;$

$\frac{C^{(2)}}{12}=\frac{a}{12 \pi^{2} G} r_{h} \frac{\alpha}{\kappa}\left(\frac{d \phi}{d r}\right)_{r_{h}}$;

$$
Q_{0}^{(2)}=\frac{a}{12 \pi^{2} G} r_{h} \frac{\kappa}{\alpha}\left(\frac{d \phi}{d r}\right)_{r_{h}} ;
$$

$\frac{C^{(3)}}{12}=-\frac{a}{2 \pi G} \frac{\alpha}{\kappa} \phi_{r_{h}} \chi ; \quad Q_{0}^{(3)}=-\frac{a}{2 \pi G} \frac{\kappa}{\alpha} \phi_{r_{h}} \chi$,

where the respective terms due to $S_{1}$ vanishes. To obtain the above expressions the explicit forms of $P^{a b c d}$, given by (19), (19), and (20), have been used in (28). $\chi$ is the twodimensional Euler characteristic of the horizon and $\phi_{r_{h}}$ is the value of $\phi$ at the horizon. Also in the above expression $A_{h}$ is the value of the quantity defined in equation (28) with $P^{a b c d}=\frac{1}{2}\left(g^{a c} g^{b d}-g^{a d} g^{b c}\right)$. Now substituting all these in the standard Cardy formula [36,37],

$S=2 \pi \sqrt{\frac{C \Delta}{6}} ; \quad \Delta \equiv Q_{0}-\frac{C}{24}$,

we find the expressions for the entropy:

$S^{E H}=\frac{A_{h}}{4 G}$,

$S^{(2)}=\frac{a}{6 \pi G} r_{h}\left(\frac{d \phi}{d r}\right)_{r_{h}}$,

$S^{(3)}=-\frac{a}{G} \phi_{r_{h}} \chi$

where we have used the identification $\alpha=\kappa$. The reason has been explained earlier below Eq. (7). This can also be taken from [35]. Hence the total expression for the entropy corresponding to the anomaly-induced action would be given by

$S_{\mathrm{tot}}=\frac{A_{h}}{4 G}+\frac{a}{6 \pi G} r_{h}\left(\frac{d \phi}{d r}\right)_{r_{h}}-\frac{a}{G} \phi_{r_{h}} \chi$.

The same expression was also obtained in $[27,28]$ by evaluating the Noether charge for a Killing vector on the horizon. The charge obtained by the authors of $[27,28]$ is on-shell. Here we derived it by the Virasoro algebra technique. The analysis adopted here is totally off-shell.

The remarkable fact about the above expression is, contrary to $[27,28]$, that the last two terms yield an additive constant to the entropy which can be neglected. This can be shown as follows. For simplicity, let us consider the classical background as the Schwarzschild metric. To obtain the explicit expression for $\phi$ in terms of metric coefficients, we need to solve Eq. (18). The solution will be found by imposing certain boundary conditions on the scalar field $\phi$. Since our only concern is the horizon we would require the finiteness of $\phi$ and its first derivative at the horizon. Such conditions lead to the following solution [26]: 


$$
\frac{d \phi}{d r}=-\frac{4 G m}{3 r(r-2 G m)} \ln \frac{r}{2 G m}-\frac{1}{2 G m}-\frac{2}{r},
$$

where $\operatorname{dilog}(x)=\int_{1}^{x} \frac{\ln (t)}{1-t} d t$. Integrating the above we find

$$
\begin{aligned}
\phi(r)= & -\frac{r}{2 G m}-2 \ln \frac{r}{2 G m}+\frac{1}{3}\left(\ln \frac{r}{2 G m}\right)^{2} \\
& +\frac{2}{3} \operatorname{dilog}\left(\frac{r}{2 G m}\right) .
\end{aligned}
$$

Note the important point that any constant term can shift the value of $\phi$ at horizon. But the respective contribution to the Noether charge and hence the entropy would then vanish since the action reduces to a topological term, which is the integral of the Euler density. Now it is easy to see that the term $\phi_{r_{h}}$ leads to a constant contribution and so the last term does not affect the Bekenstein-Hawking entropy. However, $\frac{d \phi}{d r}$ leads to a term which is proportional to the inverse of the square root of the horizon area. This would get compensated by the $r_{h}$ term. Hence the final expression for entropy leads to the usual expression of the entropy. Therefore, from the above discussion, one can conclude that the type-A trace anomaly does not lead to any correction to the entropy.

\section{Conclusions}

The idea that horizon entropy can be obtained from a certain class of diffeomorphism generators is pioneered mainly by the works of Brown, Henneaux [29] and Carlip [30]. However, all these methods use on-shell criteria, i.e., the equations of motion have been used at one stage or another. Recently, one of the authors of this paper has derived the horizon entropy using the Virasoro algebra and the Cardy formula, bypassing the use of the equation of motion [35]. In the present work, we have given an off-shell description as well, i.e., no equation of motion has been used. This again illustrates that the notion of entropy goes beyond the black hole horizon. We have started from the expressions for the Noether current and the potential for an arbitrary generally covariant Lagrangian, which could be function of metric tensor, curvature tensor, and scalar combination of different fields. From these, using the off-shell definition of the bracket among the charges [35], a general expression for the central term was derived. We then obtained the Fourier modes of the charge and the central term.

Next we have evaluated the general expressions explicitly for the anomaly-induced action which produces the type-A trace anomaly. This action has a pure geometric meaning except for the Euler characteristics and couplings appearing in it. For this particular action we have shown that the terms containing derivatives of $P^{a b c d}$ vanish in the near horizon limit and the most dominant term is the one that appears in Lanczos-Lovelock models. It has been shown that the
Fourier modes of the bracket are similar to the usual Virasoro algebra with central extension. Identifying the central charge and the zero mode eigenvalue and then using them in the Cardy formula we obtained the expression for the entropy. The result is identical to that obtained in [27,28]. Considering the classical background as the Schwarzschild metric, it has been shown that the entropy of the black hole is $A_{h} / 4 G$, the usual result. Hence the type-A trace anomaly does not lead to any correction to the horizon entropy.

So far, we have observed that there is no correction term in the entropy. Our present analysis was based on the typeA trace anomaly. As we mentioned earlier, there also exist other types of trace anomalies, like type-B etc. It would be interesting to study the effective actions corresponding to these anomalies in the present direction and investigate if they lead to any correction to the entropy. This we leave for the future.

Acknowledgments We thank T. Padmanabhan for several useful comments on the first draft of our paper. The research of one of the authors (BRM) is supported by a Lady Davis Fellowship at Hebrew University, by the I-CORE Program of the Planning and Budgeting Committee and the Israel Science Foundation (grant No. 1937/12), as well as by the Israel Science Foundation personal grant No. 24/12. The research of S.C is funded by SPM Fellowship from CSIR, Govt. of India.

Open Access This article is distributed under the terms of the Creative Commons Attribution License which permits any use, distribution, and reproduction in any medium, provided the original author(s) and the source are credited.

Funded by $\mathrm{SCOAP}^{3}$ / License Version CC BY 4.0.

\section{Appendix A: Detailed calculation for the Fourier modes of the charge and the central term}

In this appendix, using Eqs. (3) and (4) we shall obtain the expressions (6) and (7), the Fourier modes of the Noether charge and the central term. To start with, we consider a $d$ dimensional spacetime manifold $M$ with boundary given as $\partial M$, such that neighborhood of $\partial M$ admits a Killing vector $\chi^{a}$, satisfying $\chi^{2}=g_{a b} \chi^{a} \chi^{b}=0$ over the boundary $\partial M$. We shall work in a stretched horizon method as used by Carlip [30]. In this approach, all the calculations will be done on the boundary $\chi^{2}=\epsilon$ and at the end the limit $\epsilon \rightarrow 0$ will be imposed to obtain our final results. Near this stretched horizon one can define an orthogonal vector $\rho^{a}$ to $\chi^{a}$ such that

$\nabla_{a} \chi^{2}=-2 \kappa \rho_{a}$

with $\kappa$ being the surface gravity at the horizon. Next we choose the diffeomorphism vector as

$\xi^{a}=T \chi^{a}+R \rho^{a}$ 
where $R$ and $T$ are the arbitrary functions of all coordinates of spacetime. This diffeomorphism could be interpreted as deformations in the $r-t$ plane, playing a crucial role in the Euclidean approach to black hole thermodynamics [40]. The condition $\chi^{a} \chi^{b} \delta g_{a b} / \chi^{2} \rightarrow 0$, when imposed on $\chi^{a}$, leads to a relation between them:

$R=\frac{\chi^{2}}{\kappa \rho^{2}} D T$

where $D \equiv \chi^{a} \nabla_{a}$. The condition is chosen in such a way that the asymptotic Killing horizon structure remains invariant after the perturbation. The diffeomorphism formed by Eqs. (43) and (44) is said to form a closed subalgebra provided $\rho^{a} \nabla_{a} T=0$ is satisfied near the horizon. In this setup, we can define the surface element on the horizon as [35]

$d \Sigma_{a b}=d^{d-2} X \mu_{a b} ; \quad \mu_{a b}=-\frac{|\chi|}{\rho \chi^{2}}\left(\chi_{a} \rho_{b}-\chi_{b} \rho_{a}\right)$.

To evaluate the charge and the bracket we also need three identities which are valid up to $\mathcal{O}\left(\chi^{2}\right)$. The detailed derivations are given in [35] (see, Eq. (B37), Eq. (B43) and Eq. (B38)). The identities are

$$
\begin{gathered}
\nabla_{a} \xi_{b}=\frac{\chi_{a} \chi_{b}}{\chi^{2}} D T+\frac{\kappa}{\chi^{2}}\left(\chi_{a} \rho_{b}-\chi_{b} \rho_{a}\right) T \\
-\frac{1}{\kappa \chi^{2}} \chi_{a} \rho_{b} D^{2} T+R \nabla_{a} \rho_{b}, \\
P^{a b c d} \nabla_{c} \xi_{d}=P^{a b c d}\left[\frac{2 \kappa}{\chi^{2}} T-\frac{1}{\kappa \chi^{2}} D^{2} T\right] \chi_{c} \rho_{d}, \\
\nabla_{d} \nabla_{a} \xi_{b}=\frac{2 \kappa}{\chi^{4}} \chi_{a} \rho_{b} \chi_{d} D T \\
-\frac{1}{\kappa \chi^{4}} \chi_{a} \rho_{b} \chi_{d} D^{3} T-\frac{1}{\chi^{4}} \chi_{a} \chi_{b} \chi_{d} D^{2} T .
\end{gathered}
$$

We shall now use the above expressions to find the charge and the bracket. For a covariant Lagrangian, we define the bracket among the charges as

$$
\begin{aligned}
{\left[Q_{1}, Q_{2}\right] } & =\left(\delta_{\xi_{1}} Q\left[\xi_{2}\right]-\delta_{\xi_{2}} Q\left[\xi_{1}\right]\right) \\
& \equiv \int \sqrt{h} d \Sigma_{a b}\left[\xi_{2}^{a} J_{1}^{b}-\xi_{1}^{a} J_{2}^{b}\right]
\end{aligned}
$$

where the notation $J_{1}^{b}=J^{b}\left[\xi_{1}\right]$ has been used. The above expression is off-shell and free of any ambiguity. The only fact needed is that the Noether current can be expressed as the covariant derivative of an antisymmetric tensor (see Ref. [35] for more details). To evaluate it for the present setup, we first calculate the current using Eq. (3). It can be shown that the current, present in Eq. (3), takes the following form:

$$
\begin{aligned}
J^{a}= & \frac{1}{8 \pi G} P^{a b c d} \xi_{b} \xi_{c} \rho_{d} \frac{1}{\xi_{4}}\left(2 \kappa D T-\frac{1}{\kappa} D^{3} T\right) \\
& -\frac{1}{8 \pi G}\left[\frac{\kappa}{\chi^{2}}\left(\chi_{c} \rho_{d}-\chi_{d} \rho_{c}\right) T+\frac{\rho_{c} \rho_{d}}{\chi^{2}} D T-\frac{\chi_{c} \rho_{d}}{\kappa \chi^{2}} D^{2} T\right] \\
& \times \nabla_{b}\left(P^{a d b c}+P^{a c b d}\right) \\
& -\frac{1}{4 \pi G} \xi_{d} \nabla_{b} \nabla_{c} P^{a b c d} .
\end{aligned}
$$

In the intermediate steps, the identities (46), (47), and (48) have been used. Then using Eqs. (43) and (45) we obtain

$$
\begin{aligned}
& d \Sigma_{a b} \xi_{2}^{a} J_{1}^{b} \\
&=d^{d-2} X \frac{1}{32 \pi G} \frac{\rho}{|\chi|} P^{a b c d} \mu_{a b} \mu_{c d}\left(2 \kappa D T_{1}-\frac{1}{\kappa} D^{3} T_{1}\right) T_{2} \\
&+\frac{1}{8 \pi G} d^{d-2} X\left(\frac{|\chi|}{\rho \chi^{2}}\right)\left(\chi_{a} \rho_{b}-\chi_{b} \rho_{a}\right) \xi_{2}^{a} \\
& \times\left[\frac{\kappa}{\chi^{2}}\left(\chi_{c} \rho_{d}-\chi_{d} \rho_{c}\right) T_{1}\right. \\
&\left.+\frac{\rho_{c} \rho_{d}}{\chi^{2}} D T_{1}-\frac{\chi_{c} \rho_{d}}{\kappa \chi^{2}} D^{2} T_{1}\right] \nabla_{f}\left(P^{b d f c}+P^{b c f d}\right) \\
&+\frac{1}{4 \pi G} d^{d-2} X\left(\frac{\chi}{\rho \chi^{2}}\right)\left(\chi_{a} \rho_{b}-\chi_{b} \rho_{a}\right) \xi_{2}^{a} \xi_{1 d} \nabla_{f} \nabla_{c} P^{b f c d}
\end{aligned}
$$

where the relation $P^{a b c d} \mu_{a b} \mu_{c d}=\frac{4}{\rho^{2} \chi^{2}} P^{a b c d} \rho_{a} \chi_{b} \rho_{c} \chi_{d}$ has been used. Finally, substitution of Eq. (51) in Eq. (49) leads to

$$
\begin{aligned}
{\left[Q_{1}, Q_{2}\right]=} & \frac{1}{32 \pi G} \int \sqrt{h} d^{d-2} X P^{a b c d} \mu_{a b} \mu_{c d} \\
& \times\left[\frac{1}{\kappa} T_{1} D^{3} T_{2}-2 \kappa T_{1} D T_{2}\right] \\
& -\frac{1}{8 \pi G} \int \sqrt{h} d^{d-2} X \frac{|\chi|}{\rho} \frac{\chi_{b} \chi_{c} \rho_{d}}{\kappa \chi^{2}} T_{2} D^{2} T_{1} \nabla_{f} P^{b c f d} \\
& -\frac{1}{8 \pi G} \int d^{d-2} X \sqrt{h} \frac{|\chi|}{\kappa^{2} \chi^{2} \rho} \chi_{b} \chi_{c} \rho_{d} D T_{2} D^{2} T_{1} \nabla_{f} P^{b d f c} \\
& +\frac{1}{4 \pi G} \int d^{d-2} X \sqrt{h} \frac{|\chi|}{\kappa \rho^{3}} \\
& \times\left(\chi^{2} \rho_{b} \rho_{d}-\rho^{2} \chi_{b} \chi_{d}\right) T_{2} D T_{1} \nabla_{f} \nabla_{c} P^{b f c d}-(1 \leftrightarrow 2) .
\end{aligned}
$$

Next we shall obtain the expression for the charge $Q[\xi]$ in the near horizon limit. The charge is given by Eq. (5). Use of Eqs. (43) and (48) in Eq. (5) leads to

$$
\begin{aligned}
Q[\xi]= & -\frac{1}{32 \pi G} \int \sqrt{h} d^{d-2} X P^{a b c d} \mu_{a b} \mu_{c d} \\
& \times\left[2 \kappa T-\frac{1}{\kappa} D^{2} T\right] \\
& -\frac{1}{8 \pi G} \int \sqrt{h} d^{d-2} X \mu_{a b} \xi_{d}\left(\nabla_{c} P^{a b c d}\right) .
\end{aligned}
$$

Now the central term will be calculated. This is defined as

$K\left[\xi_{1}, \xi_{2}\right]=\left[Q_{1}, Q_{2}\right]-Q\left[\left\{\xi_{1}, \xi_{2}\right\}\right]$ 
where the quantity $\left[Q_{1}, Q_{2}\right]$ given in Eq. (52) and $Q\left[\left\{\xi_{1}, \xi_{2}\right\}\right]$ will be evaluated using (53) in the following way. Using Eq. (43), the Lie bracket near the horizon turns out to be

$$
\left\{\xi_{1}, \xi_{2}\right\}^{a}=\left\{T_{1}, T_{2}\right\} \chi^{a}+\left\{R_{1}, R_{2}\right\} \rho^{a}
$$

where we have $\left\{T_{1}, T_{2}\right\}=\left(T_{1} D T_{2}-T_{2} D T_{1}\right)$ and so on. Substituting this in Eq. (53) we find

$$
\begin{aligned}
& Q\left[\left\{\xi_{1}, \xi_{2}\right\}\right] \\
&=-\frac{1}{32 \pi G} \int \sqrt{h} d^{d-2} X P^{a b c d} \mu_{a b} \mu_{c d} \\
& \times\left[2 \kappa T_{1} D T_{2}-\frac{1}{\kappa} D T_{1} D^{2} T_{2}+T_{1} D^{3} T_{2}\right]-(1 \leftrightarrow 2) \\
&-\frac{1}{8 \pi G} \int \sqrt{h} d^{d-2} X \mu_{a b}\left\{\xi_{1}, \xi_{2}\right\}_{d}\left(\nabla_{c} P^{a b c d}\right) .
\end{aligned}
$$

Finally, substitution of Eqs. (52) and (56) in Eq. (54) yields the form of the central term as

$$
\begin{aligned}
K\left[\xi_{1}, \xi_{2}\right] & -\frac{1}{32 \pi G} \int \sqrt{h} d^{d-2} X P^{a b c d} \mu_{a b} \mu_{c d} \frac{1}{\kappa} D T_{1} D^{2} T_{2} \\
& -\frac{1}{8 \pi G} \int \sqrt{h} d^{d-2} X \frac{|\chi|}{\rho} \frac{\chi_{b} \chi_{c} \rho_{d}}{\kappa \chi^{2}} T_{2} D^{2} T_{1} \nabla_{f} P^{b c f d} \\
& -\frac{1}{8 \pi G} \int d^{d-2} X \sqrt{h} \frac{|\chi|}{\kappa^{2} \chi^{2} \rho} \chi_{b} \chi_{c} \rho_{d} D T_{2} D^{2} T_{1} \nabla_{f} P^{b d f c} \\
& +\frac{1}{4 \pi G} \int d^{d-2} X \sqrt{h} \frac{|\chi|}{\kappa \rho^{3}}\left(\chi^{2} \rho_{b} \rho_{d}-\rho^{2} \chi_{b} \chi_{d}\right) \\
& \times T_{2} D T_{1} \nabla_{f} \nabla_{c} P^{b f c d} \\
& -\frac{1}{4 \pi G} \int \sqrt{h} d^{d-2} X \frac{|\chi|}{\rho \chi^{2}}\left[\chi_{a} \rho_{b} \chi_{d} T_{1} D T_{2} \nabla_{c} P^{a b c d}\right. \\
& \left.+\left(\frac{\chi^{2}}{\kappa \rho^{2}}\right)^{2} D T_{1} D^{2} T_{2} \chi_{a} \chi_{b} \rho_{d} \nabla_{c} P^{a b c d}\right] \\
& -(1 \leftrightarrow 2) .
\end{aligned}
$$

Now the Fourier modes of the Noether charge and the central term will be evaluated. For this we need to first give the Fourier modes of the arbitrary function $T$. The Fourier decompositions of the functions $T_{1}$ and $T_{2}$ are taken as

$T_{1}=\sum_{m} A_{m} T_{m} ; \quad T_{2}=\sum_{n} B_{n} T_{n}$

with the reality criteria for $T_{1}$ and $T_{2}$ being given by $A_{n}^{*}=$ $A_{-n}$ and $B_{m}^{*}=B_{-m}$. Substituting Eq. (58) in Eq. (53) and using $Q[\xi]=\sum_{m} A_{m} Q_{m}$, we find the Fourier modes of the charge as given in Eq. (6). Similarly, in order to obtain the same for the central term, we define

$K\left[\xi_{1}, \xi_{2}\right]=\sum_{m, n} C_{m, n} K\left[\xi_{m}, \xi_{n}\right]$

with $C_{m, n} \equiv A_{m} B_{n}$ and so $C_{m, n}^{*} \equiv C_{-m,-n}$. This manipulation in turn leads to the Fourier decomposition of the central term as present in Eq. (7).

\section{References}

1. J.D. Bekenstein, Nuovo Cimento Lett. 4, 737 (1972)

2. J.D. Bekenstein, Phys. Rev. D 7, 2333 (1973)

3. J.D. Bekenstein, Phys. Rev. D 9, 3292 (1974)

4. J.M. Bardeen, B. Carter, S.W. Hawking, Commun. Math. Phys. 31, 161 (1973)

5. S.W. Hawking, Commun. Math. Phys. 43, 199 (1975)

6. T. Padmanabhan, Rep. Prog. Phys. 73, 046901 (2010)

7. T. Padmanabhan, J. Phys. Conf. Ser. 306, 012001 (2011)

8. W.G. Unruh, Phys. Rev. D 14, 870 (1976)

9. T. Padmanabhan, Phys. Rev. D 84, 124041 (2011). 1109.3846 [gr-qc]

10. R.M. Wald, Phys. Rev. D 48, 3427 (1993). arXiv:gr-qc/9307038

11. P. Berglund, J. Bhattacharyya, D. Mattingly, Phys. Rev. Lett. 110, 071301 (2013). arXiv:hep-th/1210.4940

12. P. Berglund, J. Bhattacharyya, D. Mattingly, Phys. Rev. D 85, 124019 (2012). arXiv:hep-th/1202.4497

13. S.W. Hawking, Commun. Math. Phys. 55, 133 (1977)

14. D.V. Fursaev, Phys. Rev. D 51, 5352 (1995). arXiv:hep-th/9412161

15. D.N. Page, New J. Phys. 7, 203 (2005). (For an extensive list of papers on logarithmic correction). arXiv:hep-th/0409024

16. R.K. Kaul, P. Majumdar, Phys. Rev. Lett. 84, 5255 (2000). arXiv:gr-qc/0002040

17. R. Banerjee, B.R. Majhi, Phys. Lett. B 662, 62 (2008). arXiv:hep-th/0801.0200

18. R. Banerjee, B.R. Majhi, JHEP 0806, 095 (2008). arXiv:hep-th/0805.2220

19. R. Banerjee, B.R. Majhi, Phys. Lett. B 674, 218 (2009). arXiv:hep-th/0808.3688

20. B.R. Majhi, Phys. Rev. D 79, 044005 (2009). arXiv:hep-th/0809.1508

21. B.R. Majhi, S. Samanta, Ann. Phys. 325, 2410 (2010). arXiv:hep-th/0901.2258

22. S. Das, S. Shankaranarayanan, S. Sur, Phys. Rev. D 77, 064013 (2008). arXiv:gr-qc/0705.2070

23. I.L. Buchbinder, S.D. Odinstov, I.L. Shapiro, Effective Action in Quantum Gravity (IOP, Bristol, 1992)

24. A.M. Polyakov, Phys. Lett. B 103, 207 (1981)

25. R. Balbinot, A. Fabbri, I.L. Shapiro, Nucl. Phys. B 559, 301 (1999). arXiv:hep-th/9904162

26. E. Mottola, R. Vaulin, Phys. Rev. D 74, 064004 (2006). arXiv:gr-qc/0604051

27. R. Aros, D.E. Diaz, A. Montecinos, JHEP 07, 012 (2010). arXiv:hep-th/1003.1083

28. R. Aros, D.E. Diaz, A. Montecinos (2013). arXiv:gr-qc/1305.4647

29. J.D. Brown, M. Henneaux, Commun. Math. Phys. 104, 207 (1986)

30. S. Carlip, Class. Quant. Grav. 16, 3327 (1999)

31. B.R. Majhi, Gen. Rel. Grav. 45, 345 (2013). arXiv:gr-qc/1210.3306

32. For further devolopments, see, B.R. Majhi, T. Padmanabhan, Phys. Rev. D 86, 101501 (2012). arXiv:gr-qc/1204.1422

33. B.R. Majhi, Adv. High Energy Phys., 386342 (2013). arXiv:gr-qc/1210.6736

34. B.R. Majhi, T. Padmanabhan, Eur. Phys. J. C 73, 2651 (2013). arXiv:1302.1206 [gr-qc]

35. B.R. Majhi, T. Padmanabhan, Phys. Rev. D 85, 084040 (2012)

36. J.A. Cardy, Nucl. Phys. B 270, 186 (1986)

37. S. Carlip, Class. Quant. Grav. 15, 3609 (1998). arXiv:hep-th/9806026

38. A. Strominger, J. High Energy Phys. JHEP 02, 009 (1998)

39. T. Padmanabhan, Gravitation: Foundations and Frontiers (Cambridge University Press, Cambridge, 2012)

40. M. Bañados, C. Teitelboim, J. Zanelli, Phys. Rev. Lett. 72, 975 (1994)

41. R. Balbinot, A. Fabbri, arXiv:gr-qc/9904034 
42. N.D. Birrell, P.C.W. Davies, Quantum Fields in Curved Space (Cambridge University Press, Cambridge, 1982)

43. T. Branson, B. Orsted, Proc. Am. Math. Soc. 113, 669 (1991)
44. S.M. Paneitz, SIGMA 4, 036 (2008)

45. T. Regge, C. Teitelboim, Ann. Phys. (N.Y.) 88, 286 (1974)

46. J. Katz, J. Bicak, D. Lynden-Bell, Phys. Rev. D 55, 5957 (1997) 